\title{
Cell-Free Prototyping of AND-Logic Gates Based on Heterogeneous RNA Activators Supplementary Information
}

François-Xavier Lehr, Maleen Hanst, Marc Vogel, Jennifer Kremer, H. Ulrich Göringer, Beatrix Suess, and Heinz Koeppl 


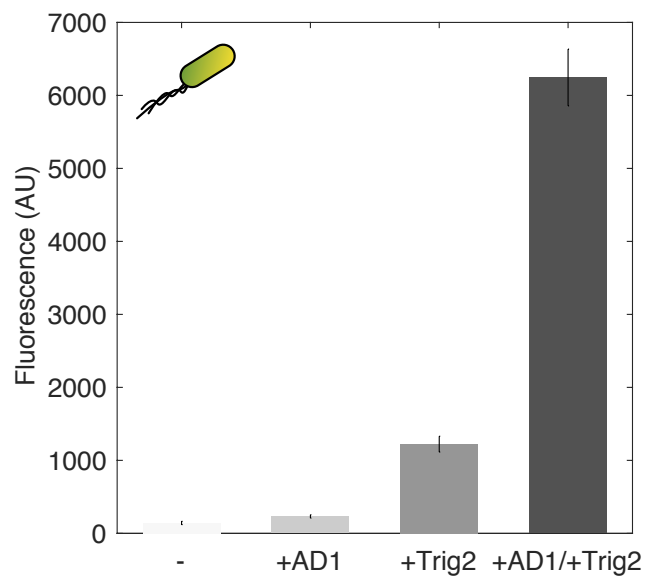

Figure 1: In vivo characterisation of the initial gate based on AD1 and toehold 2. Fluorescence endpoint measurements of the gate in top10 E. coli cells in presence $(+)$ or absence $(-)$ of the trigger/STAR DNA-encoded activators.
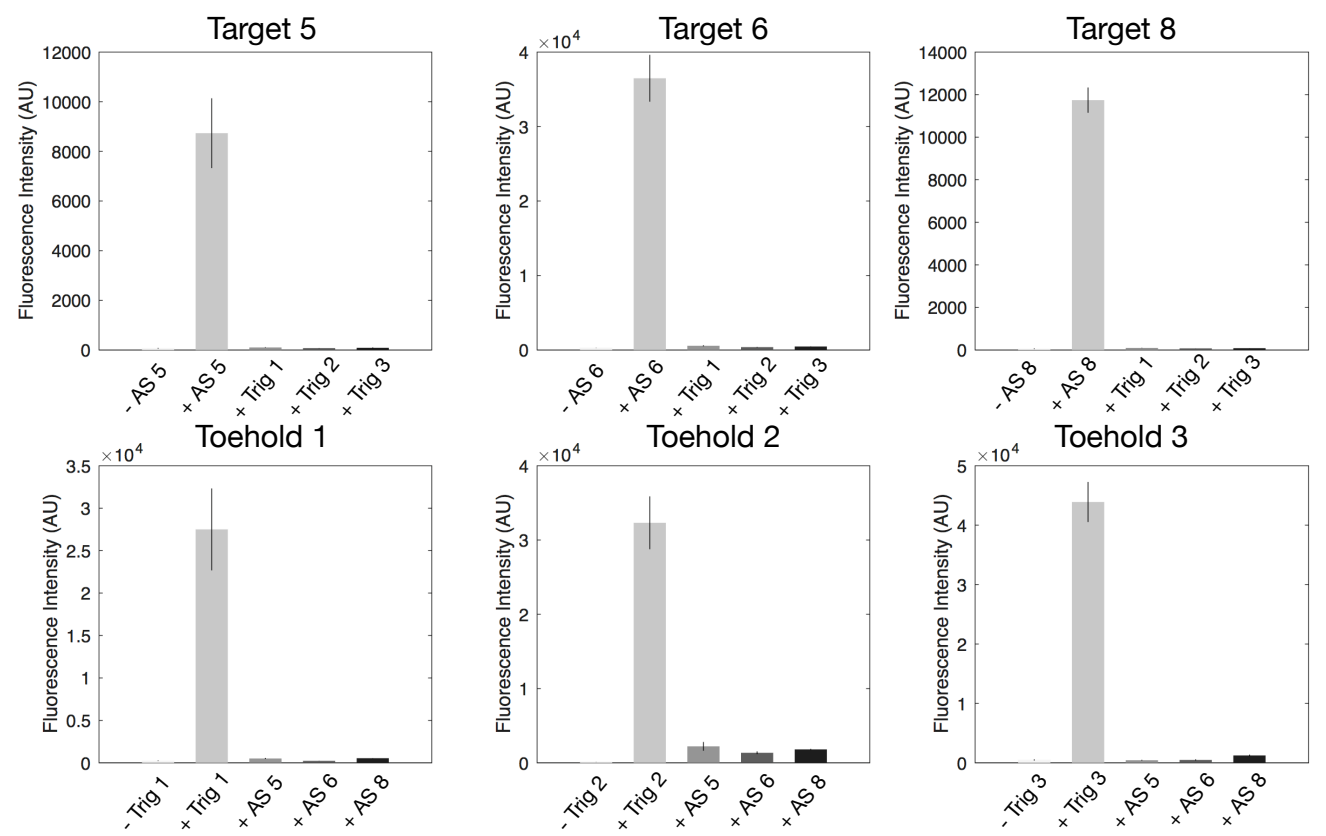

Figure 2: In vivo measurements of endpoint fluorescence for each single part, either toehold $(1,2,3)$ or STAR target $(5,6,8)$, in presence $(+)$ or absence $(-)$ of their cognate or orthogonal trigger or STAR antisense. Error bars correspond to the standard deviation of three independent measurements. 


\section{Mathematical Modeling of TX- and TL-regulated components}

We modeled the transcriptional regulation system STAR AD1-target and the translational regulation system triggertoehold 2 with parameterised ordinary differential equations (ODE). Our goal was to model the dynamics of the systems with a basic set of equations.

The TX-regulated system was modeled by the following basic reactions; the transcription and degradation of the activator RNA; the binding and unbinding of the activator RNA to the reporter mRNA; the transcription and degradation of the reporter mRNA; the translation of the reporter; and the maturation of the reporter. This resulted in the following system of ODEs

$$
\begin{aligned}
& \frac{\mathrm{d} R_{\mathrm{S}}}{\mathrm{d} t}=k_{\mathrm{S}} \cdot D_{\mathrm{S}}-d_{\mathrm{S}} \cdot R_{\mathrm{S}}, \\
& \frac{\mathrm{d} R}{\mathrm{~d} t}=k_{\mathrm{R}} \cdot\left[\frac{R_{\mathrm{S}}^{N_{\mathrm{S}}}}{\left(R_{\mathrm{S}}^{N_{\mathrm{S}}}+K_{\mathrm{S}}\right)}+p_{\mathrm{S}}\right] \cdot D_{\mathrm{G}}-d_{\mathrm{R}} \cdot R, \\
& \frac{\mathrm{d} G}{\mathrm{~d} t}=k_{\mathrm{TL}} \cdot R-\alpha \cdot G, \\
& \frac{\mathrm{d} G_{\mathrm{m}}}{\mathrm{d} t}=\alpha \cdot G,
\end{aligned}
$$

with $R_{\mathrm{S}}$ the RNA activator (STAR), and $D_{\mathrm{S}}$ the corresponding DNA template; $R$ the GFP reporter mRNA with corresponding DNA template $D_{\mathrm{G}} ; G$ the immature and $G_{\mathrm{m}}$ the mature GFP. Besides the common kinetic parameters like the transcription $\left(k_{\mathrm{S}}, k_{\mathrm{R}}\right)$, degradation $\left(d_{\mathrm{S}}, d_{\mathrm{R}}\right)$, translation $\left(k_{\mathrm{TL}}\right)$, and maturation $(\alpha)$ rates, the prefactor of the reporter transcription

$$
\left[\frac{R_{\mathrm{S}}^{N_{\mathrm{S}}}}{\left(R_{\mathrm{S}}^{N_{\mathrm{S}}}+K_{\mathrm{S}}\right)}+p_{\mathrm{S}}\right]
$$

contains a Hill coefficient, $N_{\mathrm{S}}$, the dissociation constant of the target-STAR binding, $K_{\mathrm{S}}$, and an activation probability, $p_{\mathrm{S}}$. This reflects the TX-regulation property of the system: If the activator RNA is bound to the accruing reporter mRNA, transcsription will be finished. Otherwise, there is still some activation probability for successful transcription caused by incomplete formation of the target terminator.

The ODE system for the TL-regulated system was modeled similarly by the following equations

$$
\begin{aligned}
& \frac{\mathrm{d} R_{\mathrm{T}}}{\mathrm{d} t}=k_{\mathrm{T}} \cdot D_{\mathrm{T}}-d_{\mathrm{T}} \cdot R_{\mathrm{T}}, \\
& \frac{\mathrm{d} R}{\mathrm{~d} t}=k_{\mathrm{R}} \cdot D_{\mathrm{G}}-d_{\mathrm{R}} \cdot R, \\
& \frac{\mathrm{d} G}{\mathrm{~d} t}=k_{\mathrm{TL}} \cdot\left[\frac{R_{\mathrm{T}}^{N_{\mathrm{T}}}}{\left(R_{\mathrm{T}}^{N_{\mathrm{T}}}+K_{\mathrm{T}}\right)}+p_{\mathrm{T}}\right] \cdot R-\alpha \cdot G, \\
& \frac{\mathrm{d} G_{\mathrm{m}}}{\mathrm{d} t}=\alpha \cdot G,
\end{aligned}
$$

with $R_{\mathrm{T}}$ the activator RNA (trigger), and $D_{\mathrm{T}}$ the corresponding DNA template; $R$ the GFP reporter mRNA with corresponding DNA template $D_{\mathrm{G}} ; G$ the immature and $G_{\mathrm{m}}$ the mature GFP. Similarly, $k_{\mathrm{T}}, k_{\mathrm{R}}$ denote the transcription, $d_{\mathrm{T}}, d_{\mathrm{R}}$ the degradation, $k_{\mathrm{TL}}$ the translation, and $\alpha$ the maturation rates, respectively, $N_{\mathrm{T}}$ denotes the Hill coefficient, $K_{\mathrm{T}}$ the dissociation constant of the toehold-trigger binding, and $p_{\mathrm{T}}$ the activation probability.

Both parameterised models were then calibrated to the kinetic experimental data via the MCMC method parallel tempering, described in the main text (c.f. Material and Methods), performing a Bayesian parameter inference. Within this procedure, we set $k_{\mathrm{S}}=k_{\mathrm{R}}$ and $k_{\mathrm{T}}=k_{\mathrm{R}}$ in our model, since the transcription rates within both systems should roughly be the same as the same promoters were used. 


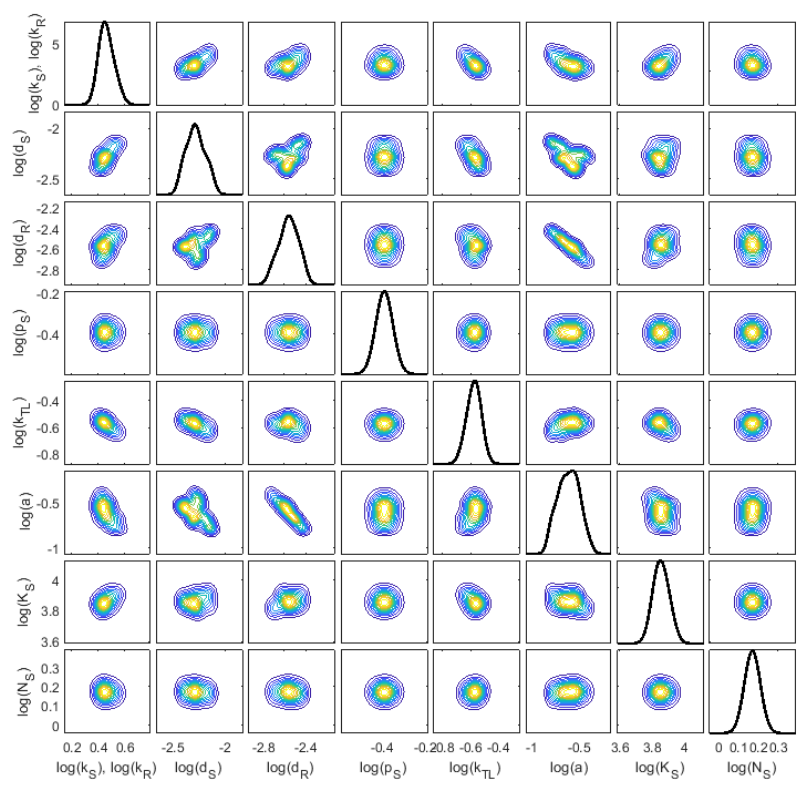

Figure 3: Parameter posterior distributions of the STAR AD1-target system; univariate distributions are on the diagonal, bivariate distributions are on the off-diagonal. The joint posterior was generated with 150000 samples in the logarithmic space. Trace plots of the parameters were considered to determine the burn-in phase and to ensure that convergence was reached.

\section{Target AD1}

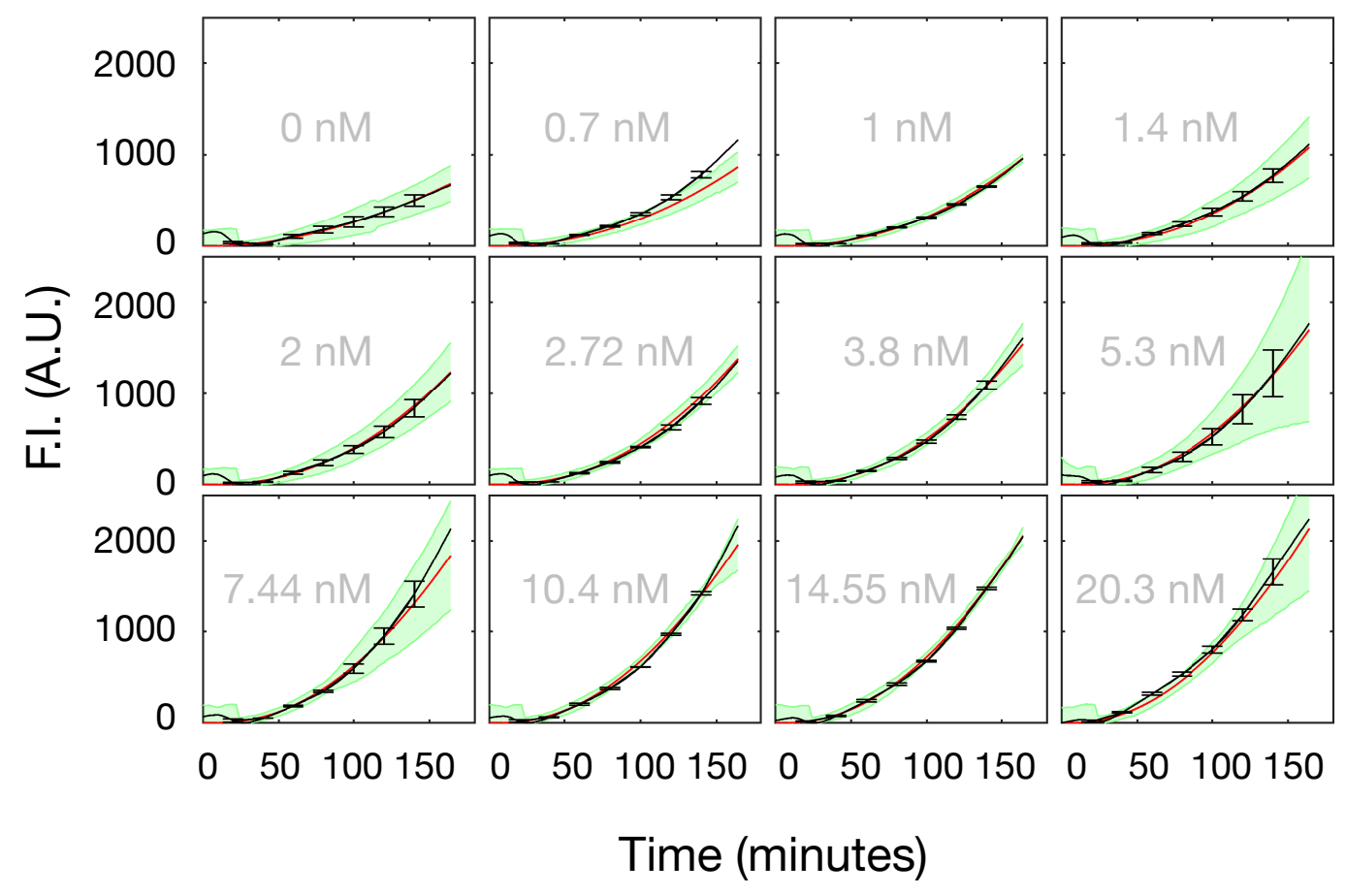

Figure 4: Calibration of single part: posterior predictive distribution of the STAR AD1-target system, for $2 \mathrm{nM}$ input concentration of target plasmid, corresponding to the posteriors in Fig. 3. Gray in the background: input concentrations of the AD1 plasmid; black: experimental data together with error bars at selected time points; red: median of parameterised model according to identified parameter posterior; green: observation model corresponding to experimentally measured standard deviation and assumption of normally distributed observation errors, 95\%and 5\%-quantiles are shown. To capture the experimental irregularities up to 25 minutes, we set our "base noise" parameter higher there. 

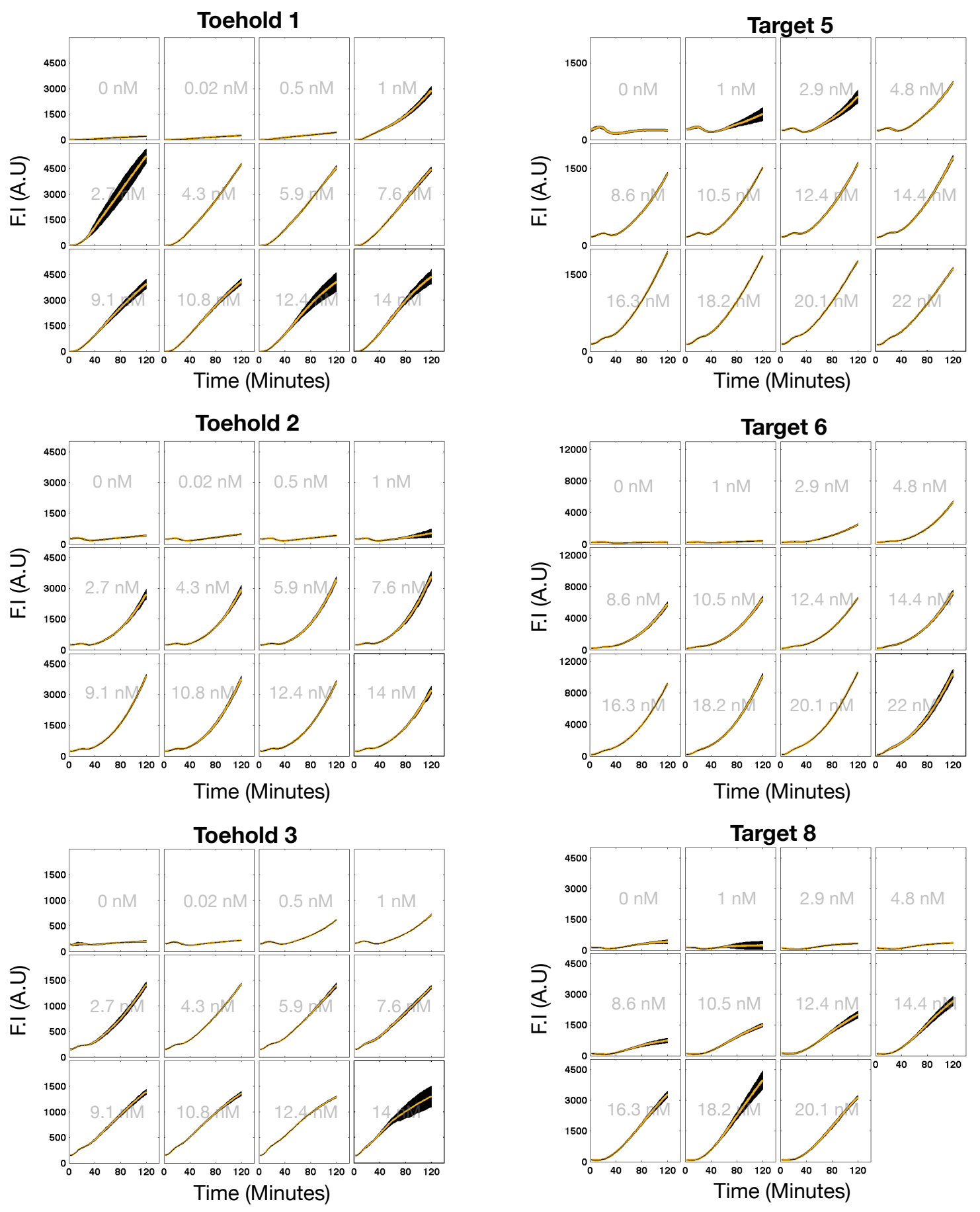

Figure 5: Kinetic characterisation of each individual part in presence of $2 \mathrm{nM}$ of reporter template, either toehold or target. Yellow lines correspond to the averaged experimentally measured fluorescence. Black shaded regions represent the experimentally measured standard deviation. Concentrations written in gray in the background correspond to the activator plasmid concentration, either trigger or STAR DNA plasmid. 
Toehold 2 with 6nM Template
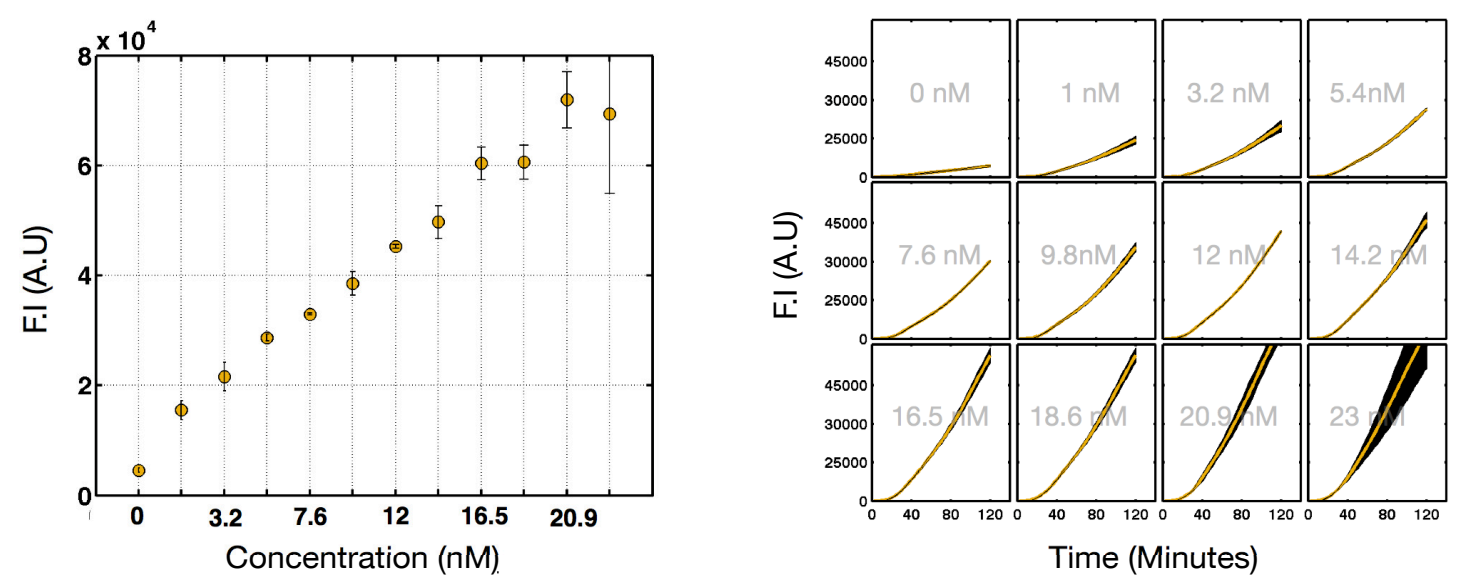

Target 6 with $6 \mathrm{nM}$ Template
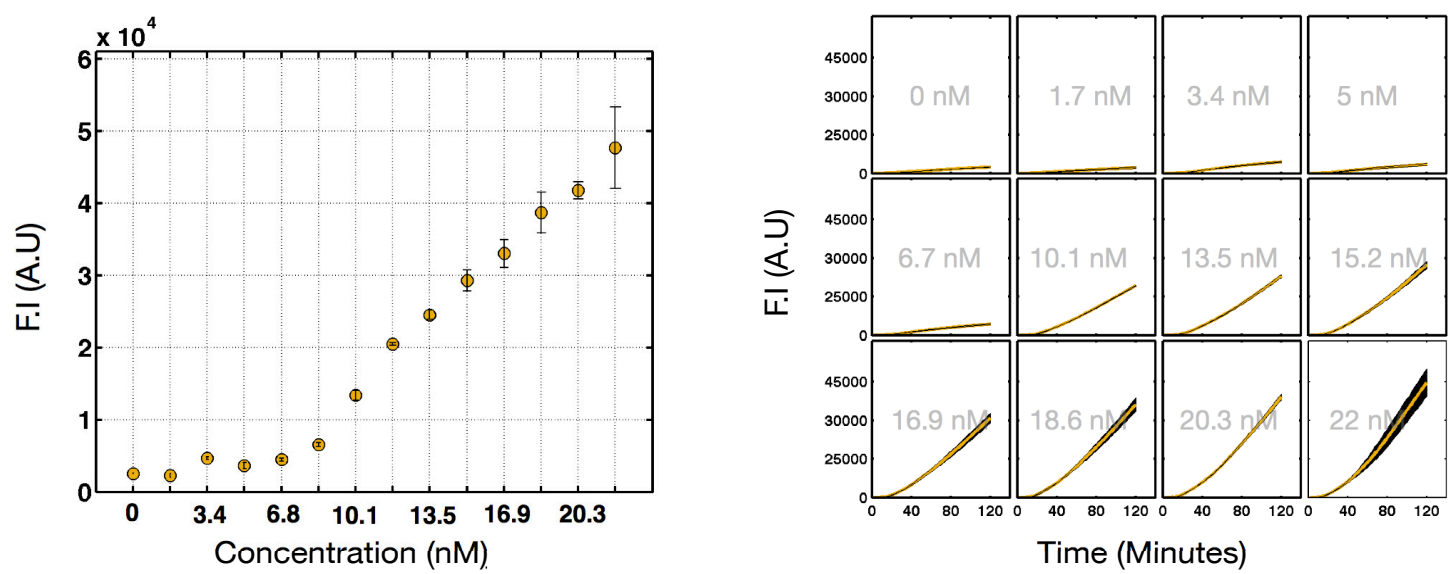

Figure 6: Kinetic characterisation of toehold 2 and target 6 parts in presence of $6 \mathrm{nM}$ of target or toehold template. Yellow lines correspond to the averaged experimentally measured fluorescence. Black shaded regions represent the experimentally measured standard deviation. Concentrations written in gray in the background correspond to the activator plasmid concentration, either trigger or STAR encoding plasmid. 
Time $=0$ minute(s)

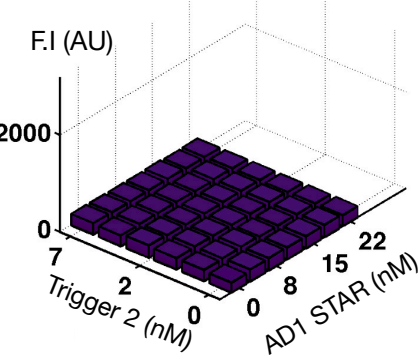

Time $=60$ minute(s)

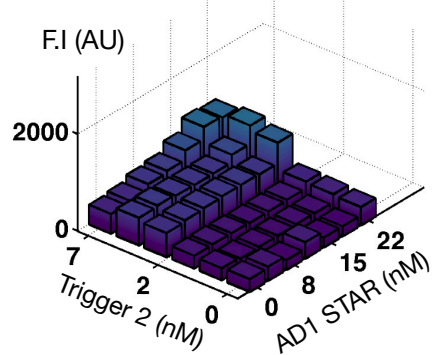

Time $=120$ minute(s)

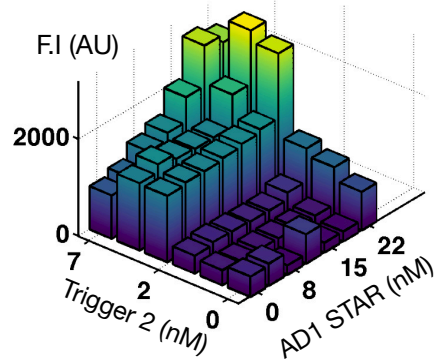

F.I (AU)

3000

2000

1000

0

Figure 7: Dose-response fluorescence measurements for the prototyping gate AD1-toehold 2 performed every 60 minutes.

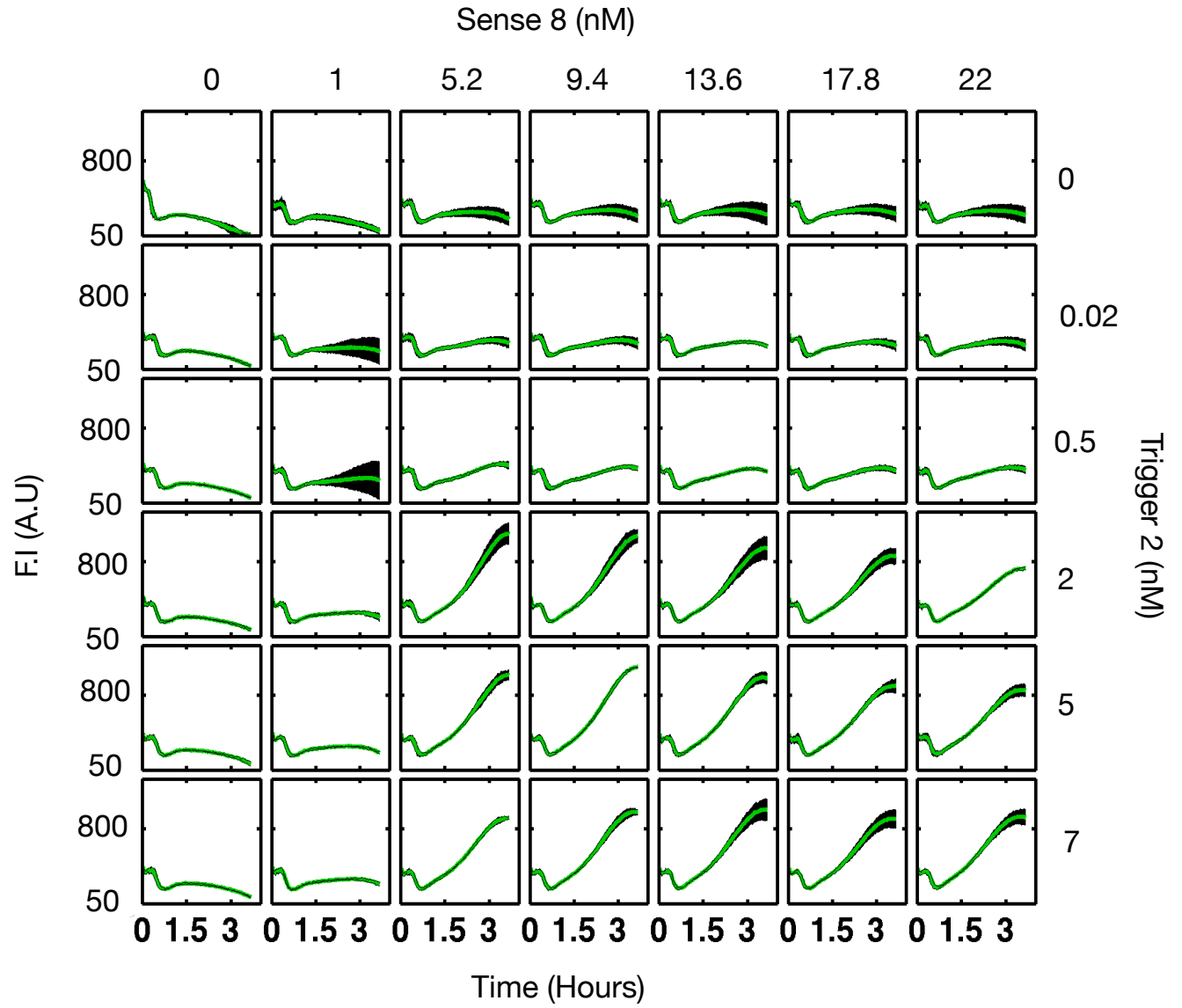

Figure 8: Concentration matrix of TX-TL time course reactions for the sense8-toehold2-SGFP gate (S8T2). Trigger and STAR encoding plasmids were simultaneously titrated from 0 to $7 \mathrm{nM}$ and 0 to $22 \mathrm{nM}$, respectively. Green lines correspond to the averaged experimentally measured fluorescence. Black shaded regions represent the experimentally measured standard deviation over three independent measurements. 
Table 1: Plasmids used.

\begin{tabular}{|c|c|c|}
\hline Plasmid Name & Plasmid architecture & Source \\
\hline pJBL2801 & $\begin{array}{c}\text { J23119 - Target AD1.S5 - RBS - sfGFP - TrrnB - } \\
\text { CmR p15A origin }\end{array}$ & $\begin{array}{l}\text { Addgene plasmid } \\
\quad \# 71207\end{array}$ \\
\hline pJBL4970 & $\begin{array}{c}\text { J23119 - Target5 - RBS - sfGFP }- \text { TrrnB }- \text { CmR } \\
\text { p15A origin }\end{array}$ & $\begin{array}{l}\text { Based on Addgene } \\
\text { plasmid \#71207 }\end{array}$ \\
\hline pJBL5816 & $\begin{array}{c}\text { J23119 - Target } 6 \text { - RBS - sfGFP }- \text { TrrnB }- \text { CmR } \\
\text { p15A origin }\end{array}$ & $\begin{array}{l}\text { Based on Addgene } \\
\text { plasmid \#71207 }\end{array}$ \\
\hline pJBL5808 & $\begin{array}{c}\text { J23119 - Target } 8 \text { - RBS }- \text { sfGFP }- \text { TrrnB }- \text { CmR } \\
\text { p15A origin }\end{array}$ & $\begin{array}{l}\text { Based on Addgene } \\
\text { plasmid \#71207 }\end{array}$ \\
\hline pJBL2807 & J23119 - STAR AD1.A5 - T500 - AmpR - ColE1 origin & $\begin{array}{l}\text { Addgene plasmid } \\
\quad \# 71203\end{array}$ \\
\hline pJBL4971 & J23119 - STAR5 - T500 - AmpR - ColE1 origin & $\begin{array}{l}\text { Based on Addgene } \\
\text { plasmid \#71203 }\end{array}$ \\
\hline pJBL5817 & J23119 - STAR6 - T500 - AmpR - ColE1 origin & $\begin{array}{l}\text { Based on Addgene } \\
\text { plasmid \#71203 }\end{array}$ \\
\hline pJBL5809 & J23119 - STAR8 - T500 - AmpR - ColE1 origin & $\begin{array}{l}\text { Based on Addgene } \\
\text { plasmid \#71203 }\end{array}$ \\
\hline pJBL-wt & J23119 - RBS - sfGFP - TrrnB - CmR p15A origin & $\begin{array}{l}\text { Based on Addgene } \\
\text { plasmid \#71207 }\end{array}$ \\
\hline pJBL-Toehold1 & $\begin{array}{c}\text { J23119 - Toehold } 1-\text { RBS }- \text { sfGFP }- \text { TrrnB }- \text { CmR } \\
\text { p15A origin }\end{array}$ & this study \\
\hline pJBL-Toehold2 & $\begin{array}{c}\text { J23119 - Toehold } 2-\text { RBS }- \text { sfGFP }- \text { TrrnB }- \text { CmR } \\
\text { p15A origin }\end{array}$ & this study \\
\hline pJBL-Toehold3 & $\begin{array}{c}\text { J23119 - Toehold } 3-\text { RBS }- \text { sfGFP }- \text { TrrnB }- \text { CmR } \\
\text { p15A origin }\end{array}$ & this study \\
\hline pJBL-trigger1 & J23119 - trigger1 - T500 - AmpR - ColE1 origin & this study \\
\hline pJBL-trigger2 & J23119 - trigger 2 - T500 - AmpR - ColE1 origin & this study \\
\hline pJBL-trigger3 & J23119 - trigger3 - T500 - AmpR - ColE1 origin & this study \\
\hline pJBL-Target AD1.S5-Toehold2 & $\begin{array}{l}\text { J23119 - Target AD1.S5 - Toehold } 2-\text { sfGFP }- \text { TrrnB - } \\
\text { CmR p15A origin }\end{array}$ & this study \\
\hline pJBL-Target5-Toehold1 & $\begin{array}{c}\text { J23119 - Target5 - Toehold } 1-\text { sfGFP }- \text { TrrnB }- \text { CmR } \\
\text { p15A origin }\end{array}$ & this study \\
\hline pJBL-Target5-Toehold2 & $\begin{array}{c}\text { J23119 - Target5 - Toehold } 2-\text { sfGFP }- \text { TrrnB }- \text { CmR } \\
\text { p15A origin }\end{array}$ & this study \\
\hline pJBL-Target5-Toehold3 & $\begin{array}{c}\text { J23119 - Target5 - Toehold } 3-\text { sfGFP }- \text { TrrnB }- \text { CmR } \\
\text { p15A origin }\end{array}$ & this study \\
\hline pJBL-Target6-Toehold1 & $\begin{array}{c}\text { J23119 - Target } 6-\text { Toehold } 1-\text { sfGFP }- \text { TrrnB }- \text { CmR } \\
\text { p15A origin }\end{array}$ & this study \\
\hline pJBL-Target6-Toehold2 & $\begin{array}{c}\text { J23119 - Target } 6-\text { Toehold } 2-\text { sfGFP }- \text { TrrnB }- \text { CmR } \\
\text { p15A origin }\end{array}$ & this study \\
\hline pJBL-Target6-Toehold3 & $\begin{array}{c}\text { J23119 - Target } 6-\text { Toehold } 3-\text { sfGFP }- \text { TrrnB }- \text { CmR } \\
\text { p15A origin }\end{array}$ & this study \\
\hline pJBL-Target8-Toehold1 & $\begin{array}{c}\text { J23119 - Target } 8-\text { Toehold } 1-\text { sfGFP }- \text { TrrnB }- \text { CmR } \\
\text { p15A origin }\end{array}$ & this study \\
\hline pJBL-Target8-Toehold2 & $\begin{array}{c}\text { J23119 - Target } 8-\text { Toehold } 2-\text { sfGFP }- \text { TrrnB }- \text { CmR } \\
\text { p15A origin }\end{array}$ & this study \\
\hline pJBL-Target8-Toehold3 & $\begin{array}{c}\text { J23119 - Target } 8-\text { Toehold } 3-\text { sfGFP }- \text { TrrnB }- \text { CmR } \\
\text { p15A origin }\end{array}$ & this study \\
\hline pJBL-STAR AD1.A5-trigger2 & $\begin{array}{c}\text { t500 - trigger } 2 \text { - J23119 - spacer - J23119 - STAR } \\
\text { AD1.A5 - T500 - AmpR - ColE1 origin }\end{array}$ & this study \\
\hline pJBL-STAR5-trigger 1 & $\begin{array}{c}\text { t500 - trigger } 1 \text { - J23119 - spacer - J23119 - STAR5 - } \\
\text { T500 - AmpR - ColE1 origin }\end{array}$ & this study \\
\hline pJBL-STAR5-trigger2 & $\begin{array}{c}\text { t500 - trigger } 2-\text { J23119 - spacer }- \text { J23119 - STAR5 - } \\
\text { T500 - AmpR - ColE1 origin }\end{array}$ & this study \\
\hline pJBL-STAR5-trigger3 & $\begin{array}{c}\text { t500 - trigger3 - J23119 - spacer - J23119 - STAR5 - } \\
\text { T500 - AmpR - ColE1 origin }\end{array}$ & this study \\
\hline pJBL-STAR6-trigger1 & $\begin{array}{c}\text { t500 - trigger1 - J23119 - spacer - J23119 - STAR6 - } \\
\text { T500 - AmpR - ColE1 origin }\end{array}$ & this study \\
\hline pJBL-STAR6-trigger2 & $\begin{array}{c}\text { t500 - trigger } 2 \text { - J23119 - spacer - J23119 - STAR6 - } \\
\text { T500 - AmpR - ColE1 origin }\end{array}$ & this study \\
\hline
\end{tabular}




\begin{tabular}{|c|c|c|}
\hline Plasmid Name & Plasmid architecture & Source \\
\hline pJBL-STAR6-trigger3 & $\begin{array}{c}\text { t500 - trigger3 - J23119 - spacer - J23119 - STAR6 - } \\
\text { T500 - AmpR - ColE1 origin }\end{array}$ & this study \\
\hline pJBL-STAR8-trigger1 & $\begin{array}{c}\text { t500 - trigger } 1 \text { - J23119 - spacer - J23119 - STAR8 - } \\
\text { T500 - AmpR - ColE1 origin }\end{array}$ & this study \\
\hline pJBL-STAR8-trigger2 & $\begin{array}{c}\text { t500 - trigger } 2-\text { J23119 - spacer - J23119 - STAR } 8 \text { - } \\
\text { T500 - AmpR - ColE1 origin }\end{array}$ & this study \\
\hline pJBL-STAR8-trigger3 & $\begin{array}{c}\text { t500 - trigger } 3 \text { - J23119 - spacer - J23119 - STAR8 - } \\
\text { T500 - AmpR - ColE1 origin }\end{array}$ & this study \\
\hline pJBL-wt(red) & J23119 - RBS - dTomato - TrrnB - CmR p15A origin & this study \\
\hline pJBL-Target6-Toehold3 & $\begin{array}{c}\text { J23119 - Target } 6 \text { - Toehold } 3 \text { - dTomato }- \text { TrrnB - } \\
\text { CmR p15A origin }\end{array}$ & this study \\
\hline
\end{tabular}


Table 2: Example Plasmids.

\begin{tabular}{|c|c|}
\hline Name & Sequence $\left[5^{\prime}->3^{\prime}\right]$ \\
\hline $\begin{array}{l}\text { pJBL- } \\
\text { STAR5- } \\
\text { trigger1 t500, } \\
\text { trigger, } \\
\text { J23119, } \\
\text { STAR, } \\
\text { ColE1, AmpR }\end{array}$ & $\begin{array}{l}\text { GAATTCaaaaaaagccegctttcggcgggctttgAGATCCctatcttatcttatctatctcgtttatccetgctttactgact } \\
\text { attgcacagaatagtcagtcccactagtattatacctaggactgagctagctgtcaaAGATCTTTAACGGGGTCAT } \\
\text { CACGGCTCATCATGCGCCAAACAAATGTGTGCAATACACGCTCGGATGACTGCA } \\
\text { TGATGACCGCACTGACTGGGGACAGCAGATCCACCTAAGCCTGTGAGAGAAGCA } \\
\text { GACACCCGACAGATCAAGGCAGTTAAATTAAAGATCTttgacagctagctcagtcctaggtat } \\
\text { aatactagttgaactgtatacattccccgcaggatagaattgaagatgaaacgatgagactgggacgaGGATC } \\
\text { caaagcccgccgaaaggcgggctttttttGGATCCTTACTCGAGTCTAGACTGCAGGCTTCCT } \\
\text { CGCTCACTGACTCGCTGCGCTCGGTCGTTCGGCTGCGGCGAGCGGTATCAGCTC } \\
\text { ACTCAAAGGCGGTAATACGGTTATCCACAGAATCAGGGGATAACGCAGGAAAGA } \\
\text { ACATGTGAGCAAAAGGCCAGCAAAAGGCCAGGAACCGTAAAAAggccgcgttgctggcg } \\
\text { tttttccacaggctccgccccctgacgagcatcacaaaaatcgacgctcaagtcagaggtggcgaaacccgacaggactataaa } \\
\text { gataccaggcgtttcccctggaagctccctcgtgcgctctcctgttccgaccetgccgcttaccggatacctgtccgcctttct } \\
\text { cccttcgggaagcgtggcgctttctcatagctcacgctgtaggtatctcagttcggtgtaggtcgttcgctccaagctgggctgt } \\
\text { gtgcacgaacccccgttcagccegaccgctgcgccttatccggtaactatcgtcttgagtccaacccggtaagacacgacttat } \\
\text { cgccactggcagcagccactggtaacaggattagcagagcgaggtatgtaggcggtgctacagagttcttgaagtggtggcctaa } \\
\text { ctacggctacactagaagaacagtatttggtatctgcgctctgctgaagccagttaccttcggaaaaagagttggtagctcttga } \\
\text { tccggcaaacaaaccaccgctggtagcggtggttttttgtttgcaagcagcagattacgcgcagaaaaaaaggatctcaagaag } \\
\text { atctttgatcttttctacggggtctgacgctcagtggaacgaaactcacgttaagggattttggtcatgaGATTATCA } \\
\text { AAAAGGATCTTCACCTAGATCCTTTTAAATTAAAAATGAAGTTTTAAATCATCT } \\
\text { AAAGTATATATGAGTAAACTTGGTCTGACAGTTAccaatgcttaatcagtgaggcacctatctcagc } \\
\text { gatctgtctatttcgttcatccatagttgcctgactccccgtcgtgtagataactacgatacgggagggcttaccatctggcccc } \\
\text { agtgctgcaatgataccgcgagacccacgctcaccggctccagatttatcagcaataaaccagccagccggaagggccgagcgca } \\
\text { gaagtggtcctgcaactttatccgcctccatccagtctattaattgttgccgggaagctagagtaagtagttcgccagttaatag }\end{array}$ \\
\hline $\begin{array}{l}\text { pJBL- } \\
\text { STAR5- } \\
\text { trigger1 t500, } \\
\text { trigger, } \\
\text { J23119, } \\
\text { STAR, } \\
\text { ColE1, AmpR }\end{array}$ & $\begin{array}{l}\text { tttgcgcaacgttgttgccattgctacaggcatcgtggtgtcacgctcgtcgtttggtatggcttcattcagctccggttcccaa } \\
\text { cgatcaaggcgagttacatgatccccatgttgtgcaaaaaagcgettagctccttcggtcctccgatcgttgtcagaagtaagt } \\
\text { tggccgcagtgttatcactcatggttatggcagcactgcataattctcttactgtcatgccatccgtaagatgcttttctgtgac } \\
\text { tggtgagtactcaaccaagtcattctgagaatagtgtatgcggcgaccgagttgctcttgcccggcgtcaatacgggataatacc } \\
\text { gcgccacatagcagaactttaaagtgctcatCATTGGAAAACGTTCTTCGGGGCGAAAACTCTCA } \\
\text { AGGATCTTACCGCTGTTGAGATCCAGTTCGATGTAACCCACTCGTGCACCCAACTG } \\
\text { ATCTTCAGCATCTTTTACTTTCACCAGCGTTTCTGGGTGAGCAAAAACAGGAAGGC } \\
\text { AAAATGCCGCAAAAAAGGGAATAAGGGCGACACGGAAATGTTGAATACTCATACTC } \\
\text { TTCCTTTTTCAATATTATTGAAGCATTTATCAGGGTTATTGTCTCATGAGCGGATA } \\
\text { CATATTTGAATGTATTTAGAAAAATAAACAAATAGGGGTTCCGCGCACATTTCCCC } \\
\text { GAAAAGTGCCACCTGACGTCTAAGAAACCATTATTATCATGACATTAACCTATAAA } \\
\text { AATAGGCGTATCACGAGGCAGAATTTCAGATAAAAAAAATCCTTAGCTTTCGCTAA } \\
\text { GGATGATTTCTG }\end{array}$ \\
\hline
\end{tabular}




\begin{tabular}{|c|c|}
\hline Name & Sequence $\left[5^{\prime}->3^{\prime}\right]$ \\
\hline $\begin{array}{c}\text { pJBL- } \\
\text { Target5- } \\
\text { Toehold1 } \\
\text { J23119, } \\
\text { Target, } \\
\text { toehold, } \\
\text { sfGFP, } \\
\text { TrrnB, CmR, } \\
\text { p15A }\end{array}$ & 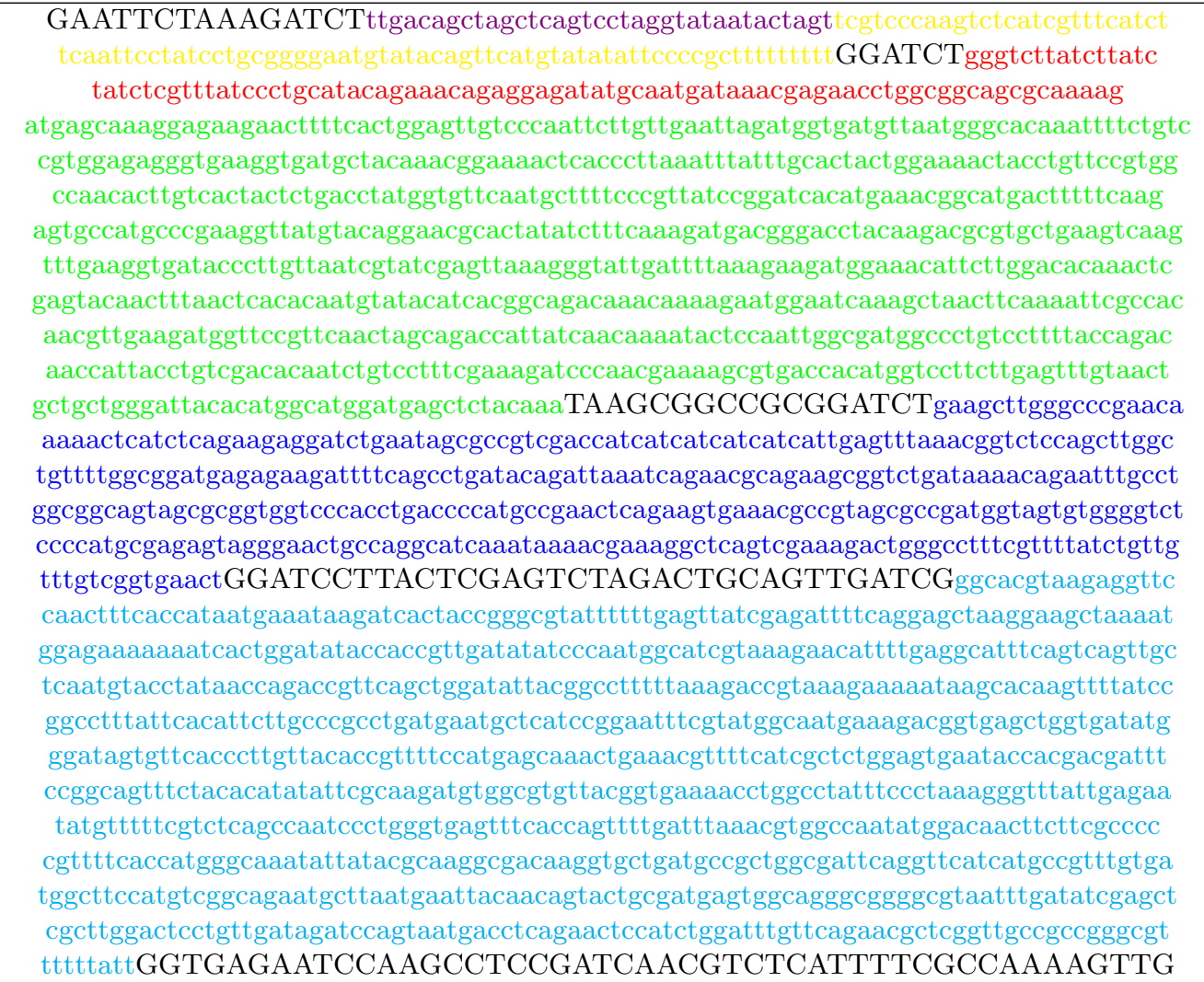 \\
\hline $\begin{array}{c}\text { pJBL- } \\
\text { Target5- } \\
\text { Toehold1 } \\
\text { J23119, } \\
\text { Target, } \\
\text { toehold, } \\
\text { sfGFP, } \\
\text { TrrnB, CmR, } \\
\text { p15A }\end{array}$ & $\begin{array}{l}\text { GCCCAGGGCTTCCCGGTATCAACAGGGACACCAGGATTTATTTATTCTGCGAAGT } \\
\text { GATCTTCCGTCACAGGTATTTATTCGGCGCAAAGTGCGTCGGGTGATGCTGCCAA } \\
\text { CTTACTGATTTAGTGTATGATGGTGTTTTTGAGGTGCTCCAGTGGCTTCTGTTTC } \\
\text { TATCAGCTGTCCCTCCTGTTCAGCTACTGACGGGGTGGTGCGTAACGGCAAAAGC } \\
\text { ACCGCCGGACATCAgcgctagcggagtgtatactggcttactatgttggcactgatgagggtgtcagtgaagtgcttc } \\
\text { atgtggcaggagaaaaaggctgcaccggtgcgtcagcagaatatgtgatacaggatatattccgcttcctcgctcactgactc } \\
\text { gctacgctcggtcgttcgactgcggcgagcggaatggcttacgaacggggcggagatttcctggaagatgccaggaagatact } \\
\text { taacagggaagtgagagggccgcggcaaagccgttttccataggctccgccccctgacaagcatcacgaaatctgacgctca } \\
\text { aatcagtggtggcgaaacccgacaggactataaagataccaggcgtttcccctggcggctccctcgtgcgctctcctgttcct } \\
\text { gcctttcggtttaccggtgtcattccgctgttatggccgcgtttgtctcattccacgcctgacactcagttccgggtaggcagt } \\
\text { tcgctccaagctggactgtatgcacgaacccccgttcagtccgaccgctgcgccttatccggtaactatcgtcttgagtccaa } \\
\text { cccggaaagacatgcaaaagcaccactggcagcagccactggtaattgatttagaggagttagtcttgaagtcatgcgccggtt } \\
\text { aaggctaaactgaaaggacaagttttggtgactgcgctcctccaagccagttacctcggttcaaagagttggtagctcagagaa } \\
\text { ccttcgaaaaaccgccetgcaaggcggttttttcgttttcagagcaagagattacgcgcagaccaaaacgatctcaagaagatc } \\
\text { atcttattaatcagataaaatattCTAGATTTCAGTGCAATTTATCTCTTCAAATGTAGCACC } \\
\text { TGAAGTCAGCCCCATACGATATAAGTTGTAATTCTCATGTTTGACAGCTTATCAT } \\
\text { CGATAAGCTTCCGATGGCGCGCCGAGAGGCTTTACACTTTATGCTTCCGGCT }\end{array}$ \\
\hline
\end{tabular}

Table 3: Part sequences

\begin{tabular}{|c|c|}
\hline Name & Sequence [5'->3'] \\
\hline Promoter J23119 & TTGACAGCTAGCTCAGTCCTAGGTATAATACTAGT \\
\hline Terminator t500 & CAAAGCCCGCCGAAAGGCGGGCTTTTTTTT \\
\hline & GAAGCTTGGGCCCGAACAAAAACTCATCTCAGAAGAGGATCTGAATAGCGC \\
Terminator TrrnB & CGTCGACCATCATCATCATCATCATTGAGTTTAAACGGTCTCCAGCTTGGC \\
& TGTTTGGCGGATGAGAGAAGATTTTCAGCCTGATACAGATTAAATCAGAA \\
& CCAGAAGCGGTCTGATAAAACAGAATTTGCCTGGCGGCAGTAGCGCGGTGG \\
& TCCACCTGACCCCATGCCGAACTCAGAAGTGAAACGCCGTAGCGCCGATG \\
& AAACGAAAGGCTCAGTCGAAAGACTGGGCCTTTCGTTTTATCTGTTGTTTG TCGGTGAACT \\
\hline
\end{tabular}




\begin{tabular}{|c|c|}
\hline Name & Sequence [5'->3'] \\
\hline \multirow{13}{*}{ AmpR } & CCAATGCTTAATCAGTGAGGCACCTATCTCAGCGATCTGTCTATTTCGTTC \\
\hline & АTCCATAGTTGCCTGACTCCCCGTCGTGTAGATAACTACGATACGGGAGGG \\
\hline & CTTACCATCTGGCCCCAGTGCTGCAATGATACCGCGAGACCCACGCTCACC \\
\hline & GGCTCCAGATTTATCAGCAATAAACCAGCCAGCCGGAAGGGCCGAGCGCAG \\
\hline & AAGTGGTCCTGCAACTTTATCCGCCTCCATCCAGTCTATTAATTGTTGCCG \\
\hline & GGAAGCTAGAGTAAGTAGTTCGCCAGTTAATAGTTTGCGCAACGTTGTTGC \\
\hline & CATTGCTACAGGCATCGTGGTGTCACGCTCGTCGTTTGGTATGGCTTCATT \\
\hline & CAGCTCCGGTTCCCAACGATCAAGGCGAGTTACATGATCCCCCATGTTGTG \\
\hline & CAAAAAAGCGGTTAGCTCCTTCGGTCCTCCGATCGTTGTCAGAAGTAAGTT \\
\hline & GGCCGCAGTGTTATCACTCATGGTTATGGCAGCACTGCATAATTCTCTTAC \\
\hline & TGTCATGCCATCCGTAAGATGCTTTTCTGTGACTGGTGAGTACTCAACCAA \\
\hline & GTCATTCTGAGAATAGTGTATGCGGCGACCGAGTTGCTCTTGCCCGGCGTC \\
\hline & AATACGGGATAATACCGCGCCACATAGCAGAACTTTAAAAGTGCTCAT \\
\hline \multirow{18}{*}{$\mathrm{CmR}$} & GGCACGTAAGAGGTTCCAACTTTCACCATAATGAAATAAGATCACTACCG \\
\hline & GGCGTATTTTTTGAGTTATCGAGATTTTCAGGAGCTAAGGAAGCTAAAAT \\
\hline & GGAGAAAAAAATCACTGGATATACCACCGTTGATATATCCCAATGGCATC \\
\hline & GTAAAGAACATTTTGAGGCATTTCAGTCAGTTGCTCAATGTACCTATAAC \\
\hline & CAGACCGTTCAGCTGGATATTACGGCCTTTTTAAAGACCGTAAAGAAAAA \\
\hline & TAAGCACAAGTTTTATCCGGCCTTTATTCACATTCTTGCCCGCCTGATGA \\
\hline & ATGCTCATCCGGAATTTCGTATGGCAATGAAAGACGGTGAGCTGGTGATA \\
\hline & TGGGATAGTGTTCACCCTTGTTACACCGTTTTCCATGAGCAAACTGAAAC \\
\hline & GTTTTCATCGCTCTGGAGTGAATACCACGACGATTTCCGGCAGTTTCTAC \\
\hline & ACATATATTCGCAAGATGTGGCGTGTTACGGTGAAAACCTGGCCTATTTC \\
\hline & CCTAAAGGGTTTATTGAGAATATGTTTTTCGTCTCAGCCAATCCCTGGGT \\
\hline & GAGTTTCACCAGTTTTGATTTAAACGTGGCCAATATGGACAACTTCTTCG \\
\hline & CCCCCGTTTTCACCATGGGCAAATATTATACGCAAGGCGACAAGGTGCTG \\
\hline & ATGCCGCTGGCGATTCAGGTTCATCATGCCGTTTGTGATGGCTTCCATGT \\
\hline & CGGCAGAATGCTTAATGAATTACAACAGTACTGCGATGAGTGGCAGGGCG \\
\hline & GGGCGTAATTTGATATCGAGCTCGCTTGGACTCCTGTTGATAGATCCAGT \\
\hline & AATGACCTCAGAACTCCATCTGGATTTGTTCAGAACGCTCGGTTGCCGCC \\
\hline & GGGCGTTTTTTATT \\
\hline \multirow{14}{*}{ ColE1 origin } & GGCCGCGTTGCTGGCGTTTTTCCACAGGCTCCGCCCCCCTGACGAGCATC \\
\hline & ACAAAAATCGACGCTCAAGTCAGAGGTGGCGAAACCCGACAGGACTATAA \\
\hline & AGATACCAGGCGTTTCCCCCTGGAAGCTCCCTCGTGCGCTCTCCTGTTCC \\
\hline & GACCCTGCCGCTTACCGGATACCTGTCCGCCTTTCTCCCTTCGGGAAGCG \\
\hline & TGGCGCTTTCTCATAGCTCACGCTGTAGGTATCTCAGTTCGGTGTAGGTC \\
\hline & GTTCGCTCCAAGCTGGGCTGTGTGCACGAACCCCCCGTTCAGCCCGACCG \\
\hline & CTGCGCCTTATCCGGTAACTATCGTCTTGAGTCCAACCCGGTAAGACACG \\
\hline & ACTTATCGCCACTGGCAGCAGCCACTGGTAACAGGATTAGCAGAGCGAGG \\
\hline & TATGTAGGCGGTGCTACAGAGTTCTTGAAGTGGTGGCCTAACTACGGCTA \\
\hline & CACTAGAAGAACAGTATTTGGTATCTGCGCTCTGCTGAAGCCAGTTACCT \\
\hline & TCGGAAAAAGAGTTGGTAGCTCTTGATCCGGCAAACAAACCACCGCTGGT \\
\hline & AGCGGTGGTTTTTTTGTTTGCAAGCAGCAGATTACGCGCAGAAAAAAAGG \\
\hline & АTCTCAAGAAGATCCTTTGATCTTTTCTACGGGGTCTGACGCTCAGTGGA \\
\hline & ACGAAAACTCACGTTAAGGGATTTTGGTCATGA \\
\hline \multirow{17}{*}{ p15A origin } & GCGCTAGCGGAGTGTATACTGGCTTACTATGTTGGCACTGATGAGGGTGT \\
\hline & CAGTGAAGTGCTTCATGTGGCAGGAGAAAAAAGGCTGCACCGGTGCGTCA \\
\hline & GCAGAATATGTGATACAGGATATATTCCGCTTCCTCGCTCACTGACTCGC \\
\hline & TACGCTCGGTCGTTCGACTGCGGCGAGCGGAAATGGCTTACGAACGGGGC \\
\hline & GGAGATTTCCTGGAAGATGCCAGGAAGATACTTAACAGGGAAGTGAGAGG \\
\hline & GCCGCGGCAAAGCCGTTTTTCCATAGGCTCCGCCCCCCTGACAAGCATCA \\
\hline & CGAAATCTGACGCTCAAATCAGTGGTGGCGAAACCCGACAGGACTATAAA \\
\hline & GATACCAGGCGTTTCCCCCTGGCGGCTCCCTCGTGCGCTCTCCTGTTCCT \\
\hline & GCCTTTCGGTTTACCGGTGTCATTCCGCTGTTATGGCCGCGTTTGTCTCA \\
\hline & TTCCACGCCTGACACTCAGTTCCGGGTAGGCAGTTCGCTCCAAGCTGGAC \\
\hline & GTATGCACGAACCCCCCGTTCAGTCCGACCGCTGCGCCTTATCCGGTAAC \\
\hline & TATCGTCTTGAGTCCAACCCGGAAAGACATGCAAAAGCACCACTGGCAGC \\
\hline & AGCCACTGGTAATTGATTTAGAGGAGTTAGTCTTGAAGTCATGCGCCGGT \\
\hline & TAAGGCTAAACTGAAAGGACAAGTTTTGGTGACTGCGCTCCTCCAAGCCA \\
\hline & GTTACCTCGGTTCAAAGAGTTGGTAGCTCAGAGAACCTTCGAAAAACCGC \\
\hline & CCTGCAAGGCGGTTTTTTCGTTTTCAGAGCAAGAGATTACGCGCAGACCA \\
\hline & AAACGATCTCAAGAAGATCATCTTATTAATCAGATAAAATATTT \\
\hline
\end{tabular}




\begin{tabular}{|c|c|}
\hline Name & Sequence $\left[5^{\prime}->3^{\prime}\right]$ \\
\hline \multirow{15}{*}{ sfGFP } & ATGAGCAAAGGAGAAGAACTTTTCACTGGAGTTGTCCCAATTCTTGTTGA \\
\hline & ATTAGATGGTGATGTTAATGGGCACAAATTTTCTGTCCGTGGAGAGGGTG \\
\hline & AAGGTGATGCTACAAACGGAAAACTCACCCTTAAATTTATTTGCACTACT \\
\hline & GGAAAACTACCTGTTCCGTGGCCAACACTTGTCACTACTCTGACCTATGG \\
\hline & TGTTCAATGCTTTTCCCGTTATCCGGATCACATGAAACGGCATGACTTTT \\
\hline & TCAAGAGTGCCATGCCCGAAGGTTATGTACAGGAACGCACTATATCTTTC \\
\hline & AAAGATGACGGGACCTACAAGACGCGTGCTGAAGTCAAGTTTGAAGGTGA \\
\hline & TACCCTTGTTAATGTATCGAGTTAAAGGGTATTGATTTTAAAGAAGATGG \\
\hline & АAACATTCTTGGACACAAACTCGAGTACAACTTTAACTCACACAATGTAT \\
\hline & ACATCACGGCAGACAAACAAAAGAATGGAATCAAAGCTAACTTCAAAATT \\
\hline & CGCCACAACGTTGAAGATGGTTCCGTTCAACTAGCAGACCATTATCAACA \\
\hline & AAATACTCCAATTGGCGATGGCCCTGTCCTTTTACCAGACAACCATTACC \\
\hline & TGTCGACACAATCTGTCCTTTCGAAAGATCCCAACGAAAAGCGTGACCAC \\
\hline & ATGGTCCTTCTTGAGTTTGTAACTGCTGCTGGGATTACACATGGCATGGA \\
\hline & TGAGCTCTACAAA \\
\hline \multirow{4}{*}{ spacer } & AGATCTTTAACGGGGTCATCACGGCTCATCATGCGCCAAACAAATGTGTG \\
\hline & CAATACACGCTCGGATGACTGCATGATGACCGCACTGACTGGGGACAGCA \\
\hline & GATCCACCTAAGCCTGTGAGAGAAGCAGACACCCGACAGATCAAGGCAGT \\
\hline & TAAATTAAAGATCT \\
\hline \multirow{2}{*}{ Target AD1.S5 } & AGTTTTTACAGTGAATTGTTTTAATTAGTTGTATAAATGTTGGAGCAGCG \\
\hline & GGGAATGTATACAGTTCATGTATATATTCCCCGCTTTTTTTTT \\
\hline \multirow{2}{*}{ Target5 } & TCGTCCCAAGTCTCATCGTTTCATCTTCAATTCCTATCCTGCGGGGAATG \\
\hline & TATACAGTTCATGTATATATTCCCCGCTTTTTTTTT \\
\hline \multirow{2}{*}{ Target6 } & CCAGTCATCAAGTCAGTCCAGTCAAAGTTTCCGTCGTTCAGCGGGGAATGT \\
\hline & ATACAGTTCATGTATATATTCCCCGCTTTTTTTTT \\
\hline \multirow{2}{*}{ Target8 } & CCATCCTCAATCTCTACCTACTCTCACTTACTCTTATCCTGCGGGGAATGT \\
\hline & ATACAGTTCATGTATATATTCCCCGCTTTTTTTTT \\
\hline \multirow{2}{*}{ Toehold1 } & GGGTCTTATCTTATCTATCTCGTTTATCCCTGCATACAGAAACAGAGGAGA \\
\hline & TATGCAATGATAAACGAGAACCTGGCGGCAGCGCAAAAG \\
\hline \multirow{2}{*}{ Toehold2 } & GGGAGTTTGATTACATTGTCGTTTAGTTTAGTGATACATAAACAGAGGAGA \\
\hline & TATCACATGACTAAACGAAACCTGGCGGCAGCGCAAAAG \\
\hline \multirow{2}{*}{ Toehold3 } & GGGATCTATTACTACTTACCATTGTCTTGCTCTATACAGAAACAGAGGAGA \\
\hline & TATAGAATGAGACAATGGAACCTGGCGGCAGCGCAAAAG \\
\hline \multirow{2}{*}{ STAR AD1.A5 } & TGAACTGTATACATTCCCCGCTGCTCCAACATTTATACAACTAATTAAAAC \\
\hline & AATTCACTGTAAAAACT \\
\hline STAR5 & TGAACTGTATACATTCCCCGCAGGATAGGAATTGAAGATGAAACGATGAGA CTTGGGACGA \\
\hline STAR6 & TGAACTGTATACATTCCCCGCTGAACGACGGAAACTTTGACTGGACTGACT TGATGACTGG \\
\hline STAR8 & TGAACTGTATACATTCCCCGCAGGATAAGAGTAAGTGAGAGTAGGTAGAGA TTGAGGATGG \\
\hline trigger 1 & $\begin{array}{c}\text { GGGACTGACTATTCTGTGCAATAGTCAGTAAAGCAGGGATAAACGAGATAG } \\
\text { ATAAGATAAGATAG }\end{array}$ \\
\hline trigger2 & $\begin{array}{c}\text { GGGACAGATCCACTGAGGCGTGGATCTGTGAACACTAAACTAAACGACAAT } \\
\text { GTAATCAAACTAAC }\end{array}$ \\
\hline trigger3 & $\begin{array}{c}\text { GGGTGATGGGACATTCCGATGTCCCATCAATAAGAGCAAGACAATGGTAAG } \\
\text { TAGTAATAGATAAG }\end{array}$ \\
\hline SgRNA1 & tcctaggtataatactagttgtagtgacaagtgttggccaGTTTTAGAGCTAGAAATAGCAAGTTAAAATAAGGCTAGT \\
\hline SgRNA2 & gtagtgacaagtgttggccaGTTTTAGAGCTAGAAATAGCAAGTTAAAATAAGGCTAGTCCGTTATCAACTI \\
\hline SgRNA3 & $\begin{array}{c}\text { GGGACAGATCCACTGAGGCGTGGATCTGTGAACACTAAACTAAACGACAAT } \\
\text { GTAATCAAACTAAC }\end{array}$ \\
\hline SgRNA4 & $\begin{array}{c}\text { GGGACAGATCCACTGAGGCGTGGATCTGTGAACACTAAACTAAACGACAAT } \\
\text { GTAATCAAACTAAC }\end{array}$ \\
\hline Csy4 hairpin & GTTCACTGCCGTATAGGCAGCTAAGAAA \\
\hline
\end{tabular}


A

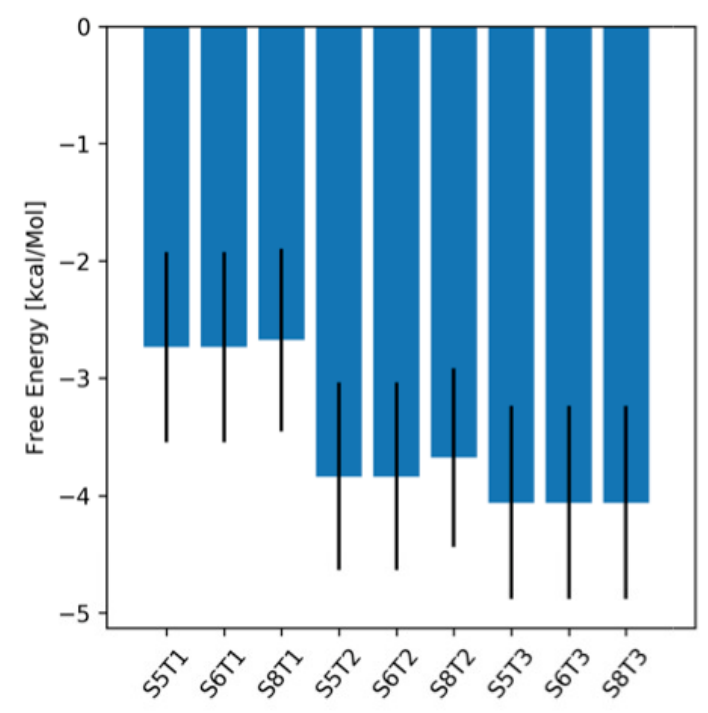

B

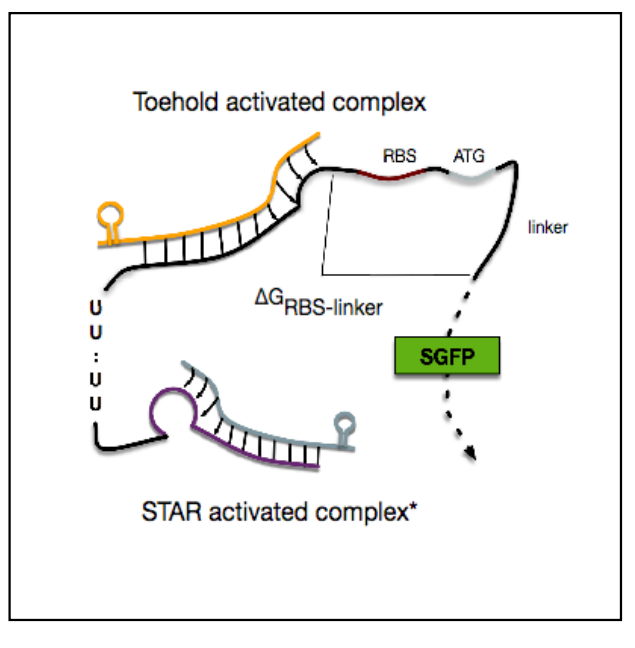

Figure 9: Free Energy of the complexes RBS-linker regions. (A) Mean Free Energies of the RBS-linker regions for a range of suboptimal structures. All suboptimal structures in an energy gap of $\Delta G=2.5 \mathrm{kCal} / \mathrm{Mol}$ have been computed with NUPACK's subopt method. We excluded all structures where the toehold-trigger complex covers at least one RBS-nucleotide; the means are calculated over the remaining suboptimal structures. Error bars: one standard deviation. (B) Graphical representation displaying the RBS-linker region for which the Free Energies are shown. 
Table 4: Minimum Free Energy (MFE) structures of the tails of all target-toehold constructs in presence of the cognate trigger activator. First lines are structures in dot-parenthesis notation, second lines show the sequences and the third lines the functional elements of interest (R: Ribosome Binding Sites; ATG: start codons). MFE structures have been computed for the compound consisting of the toehold-trigger and the complex starting from the first nucleotide downstream of the STAR-activated complex using NUPACK's subopt method. The tail of a STARtoehold sequence is here defined as the 3' part of the construct RNA downstream of the last bond to the trigger RNA, corresponding to the "RBS-linker" (see Supp. Fig. 9 B).

\begin{tabular}{|c|}
\hline $\begin{array}{l}\text { S8T3 } \\
\ldots \ldots \ldots \ldots(((\ldots(((\ldots \ldots \ldots \ldots))) \ldots))) \cdot(((\ldots \ldots))) \ldots \ldots \\
\text { ATACAGAAACAGAGGAGATATAGAATGAGACAATGGACCTGGCGGCAGCGCAAAGG } \\
------- \text { RRRRRRRR------ATG------------------ }\end{array}$ \\
\hline 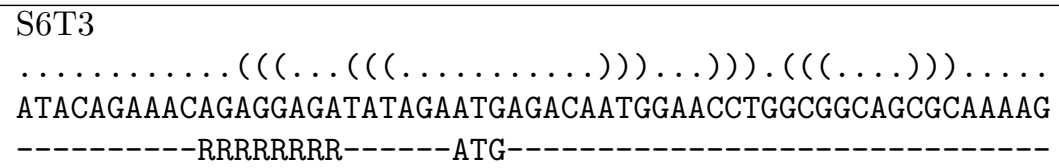 \\
\hline 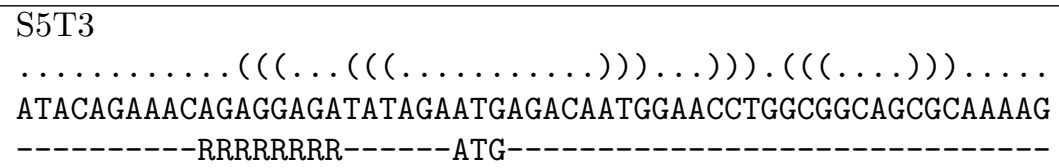 \\
\hline 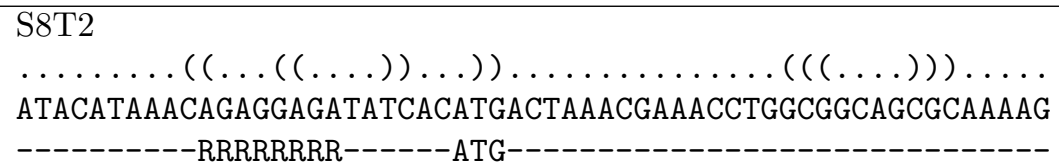 \\
\hline 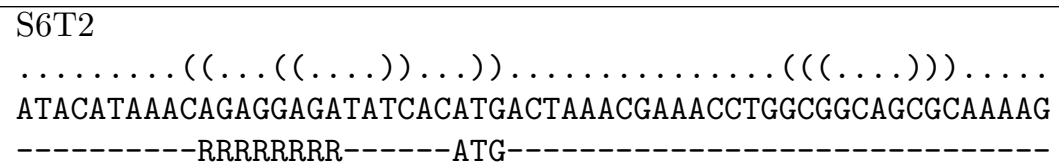 \\
\hline 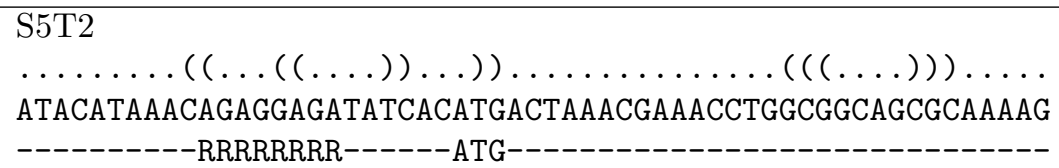 \\
\hline 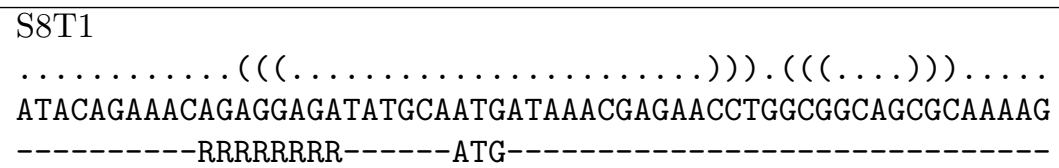 \\
\hline $\begin{array}{l}\text { S6T1 } \\
\ldots \ldots \ldots \ldots(((\ldots \ldots \ldots \ldots \ldots \ldots \ldots \ldots))) .(((\ldots \ldots))) \ldots \ldots \\
\text { ATACAGAAACAGAGGAGATATGCAATGATAAACGAGAACCTGGCGGCAGCGCAAAG } \\
--------- \text { RRRRRRRR------ATG------------------------------ }\end{array}$ \\
\hline $\begin{array}{l}\text { S5T1 } \\
\ldots \ldots \ldots \ldots(((\ldots \ldots \ldots \ldots \ldots \ldots \ldots))) .(((\ldots \ldots))) \ldots \ldots \\
\text { ATACAGAAACAGAGGAGATATGCAATGATAAACGAGAACCTGGCGGCAGCGCAAAG } \\
--------- \text { RRRRRRRR------ATG----------------------------- }\end{array}$ \\
\hline
\end{tabular}

